\title{
Le Fort I osteotomy facilitated nasotracheal intubation in a patient with concha bullosa and septal deviation: a case report
}

\author{
Je Jin Lee ${ }^{1}$, Geun Joo Choi ${ }^{1}$, Hyun Kang ${ }^{1}$, Chong Wha Baek ${ }^{1}$, Su Hyun Seo ${ }^{1}$, \\ Young-Jun Choi ${ }^{2}$, Ui-Lyong Lee ${ }^{3}$, Yong Hun Jung ${ }^{1, *}$
}

\author{
${ }^{1}$ Department of Anesthesiology and \\ Pain Medicine, Chung-Ang University \\ College of Medicine, 84, Heukseok-ro, \\ Dongjak-gu, 06974 Seoul, Republic of \\ Korea \\ ${ }^{2}$ Department of Oral \& Maxillofacial \\ Surgery, Chung-Ang University College \\ of Medicine, 84, Heukseok-ro, \\ Dongjak-gu, 06974 Seoul, Republic of \\ Korea \\ ${ }^{3}$ Department of Oral \& Maxillofacial \\ Surgery, Dental Center, Chung-Ang \\ University Hospital, 102, Heukseok-ro, \\ Dongjak-gu, 06973 Seoul, Republic of \\ Korea
}

\section{*Correspondence}

pistis23@cau.ac.kr

(Yong Hun Jung)

\begin{abstract}
Introduction: Nasotracheal intubation (NTI) is preferred for general anesthesia in maxillofacial surgery. However, NTI is often traumatic or even unsuccessful, particularly in patients with a narrow nasal pathway. In this case report, we describe a less traumatic NTI approach using maxillary downfracture of Le Fort I osteotomy.

Case presentation: A 19-year-old woman was admitted with a skeletal Class III malocclusion and scheduled to undergo bimaxillary orthognathic surgery. A preoperative evaluation revealed no other medical history and abnormal laboratory findings. Preoperative computed tomography showed nasal septal deviation, concha bullosa, and turbinate hypertrophy. A nasal Ring-Adair-Elwyn endotracheal tube and a tube exchanger could not be inserted via NTI because of her narrow nasal cavity. An oral intubation was performed temporarily and surgery was started. After a maxillary downfracture was performed, which made the nasal cavity wider than before, NTI was successfully conducted without difficulty. The patient was ventilated without any problems, and the operation was continued. Postoperatively, the patient had no further complications and her vital parameters were all stable.

Conclusions: This case report suggests that NTI after maxillary downfracture of Le Fort I osteotomy can be a good alternative that can be successfully performed with less trauma in patients undergoing orthognathic surgery who are preoperatively evaluated as having a narrow nasal cavity.
\end{abstract}

\section{Keywords}

Nasotracheal intubation; Maxillary downfracture; Orthognathic surgery; Case report

\section{Introduction}

Nasotracheal intubation (NTI) is a technique in which an endotracheal tube is inserted into the larynx through the nostril, nasal cavity, and nasopharynx. NTI is used for general anesthesia in intraoral, oropharyngeal, and maxillofacial surgeries because it provides a good surgical approach and operative view [1]. Moreover, the patient's occlusal state can be checked at any time during the operation. Despite these advantages, NTI is potentially more traumatic than orotracheal intubation and presents a higher risk of bleeding. It can also be more difficult, especially in patients with anatomical variations and turbinate hypertrophy that result in narrow nasal passages. Several methods have been reported to reduce the bleeding and trauma risk of NTI [2-7].

However, if all such methods fail, repeated unsuccessful NTI attempts to pass through narrow nasal cavity can increase the risk of damage and cause edema of nasal mucosa, putting nasal intubation procedure into a vicious cycle that makes it even more difficult. To help avoid this vicious cycle and per- form intubation successfully, we suggest a new NTI approach using maxillary downfracture of Le Fort I osteotomy. This approach was successfully used in our patient who could not be intubated through both the nostrils by any other method because of a narrow nasal cavity.

\section{Case presentation}

A 19-year-old woman (height, $165 \mathrm{~cm}$; weight, $54 \mathrm{~kg}$ ) with a skeletal Class III malocclusion was scheduled to undergo bimaxillary orthognathic surgery including Le Fort I osteotomy, bilateral sagittal split ramus osteotomy, and genioplasty. A preoperative evaluation revealed that her American Society of Anesthesiologists (ASA) physical status was class I, with no other medical history and abnormal laboratory findings, except sinus arrhythmia on electrocardiography. She had no history of facial deformity or serious facial trauma or surgery. On physical examination, her airway evaluation including thyromental distance and neck circumference was unremarkable. She had no limitation of neck range of motion and mouth opening. A 


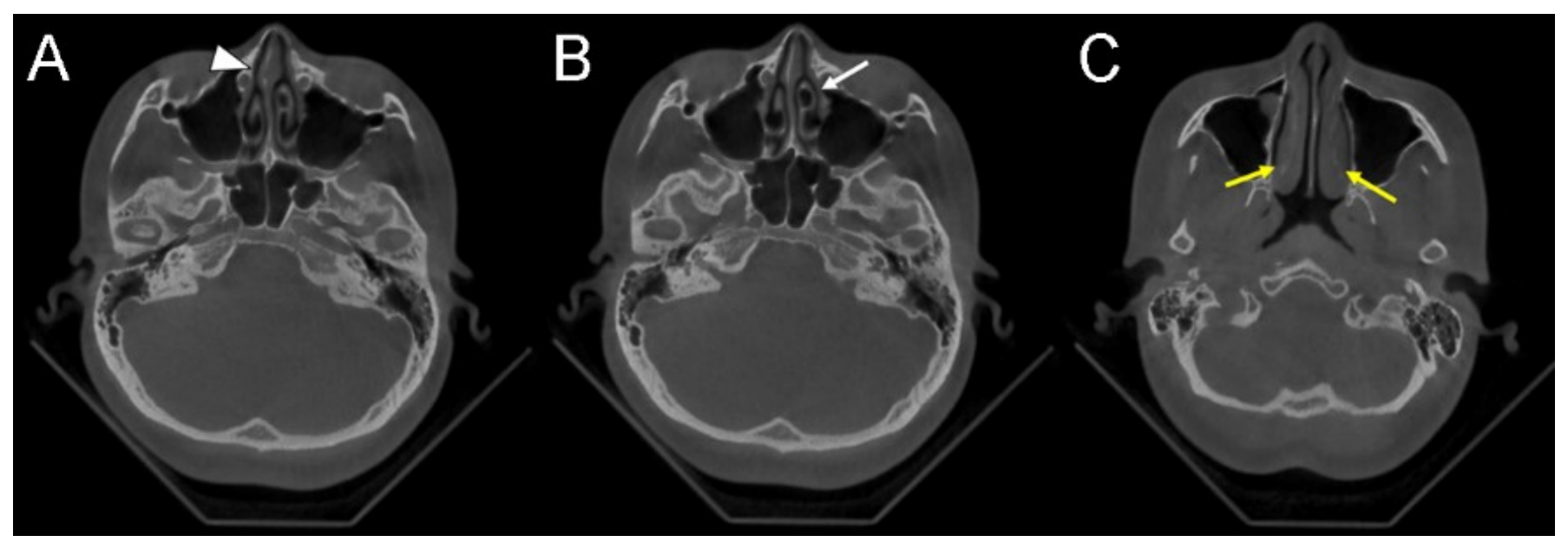

F I G U R E 1. The patient's preoperative dental cone beam computed tomography axial image. (A) Nasal septal deviation to the right (white arrowhead). (B) Concha bullosa of the left middle turbinate (white arrow). (C) Inferior turbinate hypertrophy (yellow arrows).

dental cone beam computed tomography (CT) showed rightsided nasal septal deviation, concha bullosa of the left middle turbinate, and inferior turbinate hypertrophy (Fig. 1). A nasal endoscopy was not included in preoperative evaluation in this case.

In the operating room, standard monitoring devices were used including a pulse oximeter, electrocardiography device, and non-invasive blood pressure monitor. Preoxygenation was performed for 5 minutes using a face mask with $100 \%$ oxygen. Anesthesia was induced with intravenous fentanyl $(100 \mathrm{mcg})$, $2 \%$ lidocaine $(40 \mathrm{mg})$, and propofol $(100 \mathrm{mg})$. Neuromuscular blockade was achieved by intravenous administration of rocuronium (40 $\mathrm{mg})$, and manual bag-mask ventilation was easily accomplished.

Intubation was first attempted with a lubricated nasal RingAdair-Elwyn endotracheal tube (nasal RAE tube) with an internal diameter (ID) of $6 \mathrm{~mm}$ that had been softened in warm water. However, the intubation was unsuccessful because of nasal passage blockages on both sides. After further oxygenation, intubation was attempted again via the Seldinger technique using an endotracheal tube exchanger as a guide wire. A 14-Fr endotracheal tube exchanger was inserted through the patient's nostril. Although the tube exchanger had a much smaller diameter ( $\sim 4.67 \mathrm{~mm})$ when compared to the nasal RAE tube, the patient's nasal cavity was still too narrow for the tube exchanger to pass through. As a result, the second NTI attempt was also unsuccessful.

While maintaining ventilation by mask bagging with $100 \%$ oxygen and 5\% sevoflurane, the anesthesiologist and operator decided to perform orotracheal intubation and re-approach the nasal cavity after the maxillary downfracture procedure, which is a part of Le Fort I osteotomy. As there were no other factors suggesting difficult intubation except the narrow nasal cavity shown in CT findings, oral intubation was performed easily with a reinforced tube with an ID of $7 \mathrm{~mm}$, and anesthesia was maintained with $6 \%$ desflurane and a $50 \% \mathrm{O}_{2} / 50 \% \mathrm{~N}_{2} \mathrm{O}$ mixture.

After 25 minutes, maxillary downfracture and additional partial removal of the nasal septum and concha crest were performed. As a result, the vertical dimension of the nasal passage increased. Oral and tracheal suctions were performed, and sufficient oxygenation was achieved before changing the endotracheal tube. A nasal RAE tube with an ID of $6 \mathrm{~mm}$ was passed through the right side of the widened nasal cavity without any difficulty. After securing the laryngoscopic view, the orotracheal tube was removed, and the nasal RAE tube was advanced through the vocal cord immediately (Fig. 2). After the procedure, tracheal suction through the tube showed minimal blood-tinged secretion. The patient was ventilated without any problems, and the operation was continued.

At the end of the surgery, sugammadex (200 mg) was administered for reversal of the rocuronium-induced neuromuscular block. Extubation was performed safely when the patient was fully awake and able to obey commands. The patient had no further complications and her vital parameters were all stable. She was postoperatively transferred to the general ward and discharged after 5 days without any sequelae. Institutional Review Board (IRB) of Chung-Ang University Hospital approved this case report (IRB number: 2101-01119351). The patient provided written informed consent for the publication of this report.

\section{Discussion}

For orthognathic surgery, NTI is an essential choice for favorable surgical approach and better outcomes. However, intubation through the nasal cavity can lead to trauma including epistaxis, submucosal laceration, and turbinate avulsion [1,8]. This is more likely to occur with the use of oversized tubes, use of excessive force, or repeated unsuccessful attempts. In consideration of the possibility of postoperative airway obstruction and dyspnea in patients undergoing orthognathic surgery, traumatic NTI should be avoided [9]. Furthermore, it may cause blood aspiration and airway edema in the postoperative period [1].

To perform a non-traumatic NTI, an understanding of the basic anatomy and identification of the patient's anatomical variations of the nasal passage are essential. For assessing 


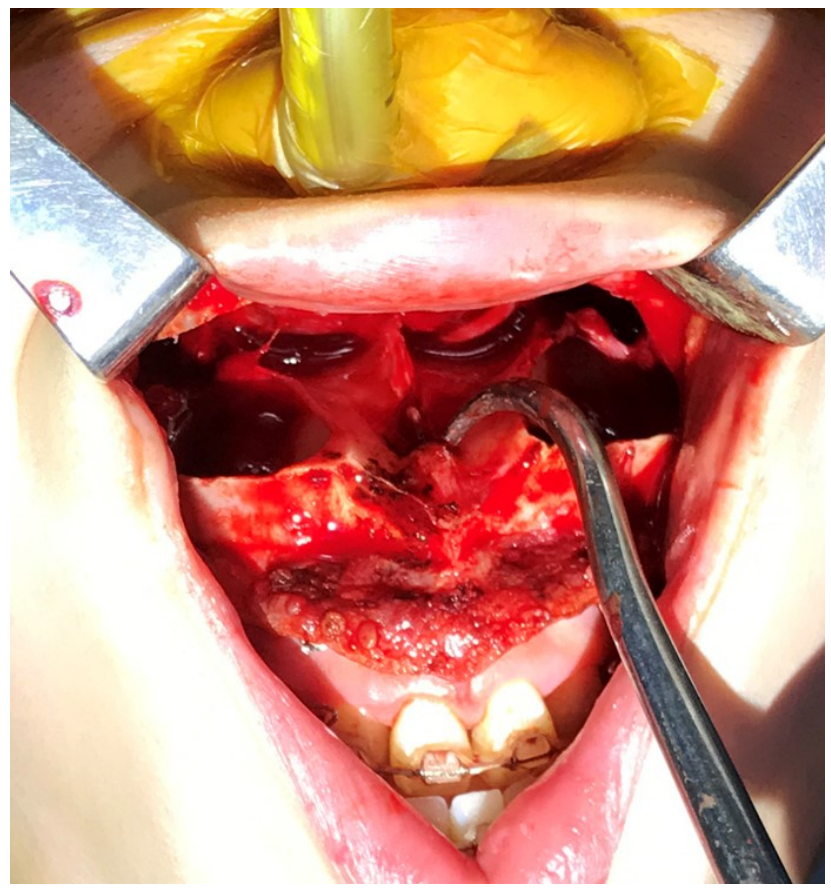

F I G U RE 2. Intraoperative clinical photograph of nasotracheal intubation (NTI) after maxillary downfracture. NTI via a nasal Ring-Adair-Elwyn tube with an internal diameter of $6 \mathrm{~mm}$ was successful after maxillary downfracture.

the patient's nasal anatomy, both nasal endoscopy and CT can be used $[1,10]$. For the patient in our case, nasal intubation was expected to be difficult because of the variation in the anatomical structure of her nose as shown by the preoperative cone beam CT. Anatomical variations, including nasal septum deviation, concha bullosa, and turbinate hypertrophy leading to nasal cavity narrowing, can interrupt the movement of the tube through the nasal passage [1]. There were no other factors found that could be expected to be difficult for intubation in our patient.

Various nasal intubation techniques that can be used in difficult situations have been reported to minimize trauma. The most fundamental approach is proper tube preparation. A suitable tube size that is small enough to pass through the nasal passage and large enough to allow sufficient ventilation should be chosen. Thermosoftening and lubrication of the nasotracheal tube can reduce epistaxis and nasal damage. Pretreatment with topical vasoconstrictors is also a widespread method $[1,7]$. Incremental mechanical dilation of the nasal passage has been proposed to decrease trauma. However, this method may cause repeated trauma and more bleeding [11]. Sheathing of the distal tip of the endotracheal tube with a red rubber catheter has been reported to reduce trauma and prevent impaction of tissue by the end of the tube $[2,5]$. The use of an endotracheal tube introducer (bougie) or a redrubber catheter to guide the advancement of a tube has been reported as a more successful and less-traumatic technique $[3,4]$. Submucosal inferior turbinectomy prior to orthognathic surgery can be considered when these noninvasive methods have been unsuccessful [10].

In our case, intubation after thermosoftening and lubrication of the tube was unsuccessful. We did not choose a thinner tube because of the concern that increasing resistance to ventilation would be unfavorable during the operation time which were expected to be about 3 hours. The insertion of the endotracheal tube exchanger was unsuccessful although it had a smaller ID than the nasal RAE tube; therefore, we could not try the Seldinger technique. One method we missed was using flexible fiberoptic bronchoscope. As fiberoptic bronchoscope is not only an intubating device but also a diagnostic tool, it could have provided us useful information for identifying patient's nasal structures [1]. Considering that the RAE tube passes through nasal cavity by pressing the surrounding soft tissue, the failure of NTI attempts using conventional methods was unpredictable based on $\mathrm{CT}$ findings only. Because there were no other factors suggesting difficult intubation, we decided to implement oral intubation as an alternative and planned changing to nasal intubation after maxillary downfracture.

The final procedure performed in our case using maxillary downfracture of Le Fort I osteotomy can be a good alternative for patients undergoing orthognathic surgery who have narrow nasal cavities. A major advantage of this technique is that non-traumatic intubation is possible even when other methods described above are unsuccessful. Because maxillary downfracture involves the bilateral maxillary buttresses and separates the nasal septum from the maxilla, it allows for further dissection of the nasal floor and nasal mucosa [12]. According to this anatomical knowledge, we expected the nasal pathway to become wider and free from obstacles. This may seem to be more invasive than previously studied methods; however, it does not require any further incisions in comparison to the previously planned operative procedures. In some patients who have been indicated for inferior turbinectomy due to turbinate hypertrophy with nasal airway obstruction, elective partial inferior turbinectomy can be performed simultaneously using a safe method with minimal complications [13].

A disadvantage of this technique is the risk of aspiration of the blood coming from the surgical site while changing the tube. Furthermore, because of the blood and secretion, laryngoscopic view might be compromised during NTI. To reduce the risk of aspiration and secure the laryngoscopic view, sufficient hemostasis and oral suction must be performed beforehand. In order to maintain the patient's hemodynamic stability during the procedure, proper level of neuromuscular block and anesthesia depth are needed. As this intra-operative technique is not free from infection issue, it needs to be implemented asepticly as much as possible. Because of the complexity of the suggested method, the clear communication and close cooperation between the anesthesiologist and surgeon are highly required.

One study that surveyed 378 patients who underwent bimaxillary orthognathic surgery reported that nasal congestion was the worst postoperative symptom that the patients complained of [14]. Disorganized and excessive NTI attempts may irritate the nasal mucosa and cause airway edema. It may worsen the patient's nasal congestion and result in difficult postoperative airway management, especially in patients with narrow nasal cavities. To prevent deterioration in these patients, our new NTI approach using maxillary downfracture should be considered based on a preoperative evaluation of the patient's nasal structure. 


\section{Conclusions}

In conclusion, we recommend our NTI technique using maxillary downfracture of Le Fort I osteotomy, which results in less trauma, particularly in patients undergoing orthognathic surgery who have been evaluated as having a narrow nasal cavity. It can be a good alternative when other techniques are unsuccessful.

\section{AUTHOR CONTRIBUTIONS}

YHJ and YJC designed the study. JJL, HK, SHS, and YJC conducted investigation and analyzed the data. JJL, GJC wrote the original draft. JJL, GJC, CWB, and UL reviewed and revised the manuscript. YHJ supervised the study. All authors approved the final manuscript.

\section{ETHICS APPROVAL AND CONSENT TO PARTICIPATE}

This study has been approved by the Institutional Review Board of Chung-Ang University Hospital (IRB number: 2101011-19351). Written informed consent was obtained from the patient for the publication of this case report.

\section{ACKNOWLEDGMENT}

No assistance in the preparation of this article is to be declared.

\section{FUNDING}

This research received no external funding.

\section{CONFLICT OF INTEREST}

The authors declare no conflict of interest.

\section{REFERENCES}

[1] Prasanna D, Bhat S. Nasotracheal intubation: an overview. Journal of Maxillofacial and Oral Surgery. 2014; 13: 366-372.
[2] Ray TL, Tobias JD. An alternative technique for nasotracheal intubation. Southern Medical Journal. 2003; 96: 1039-1041.

[3] Abrons RO, Vansickle RA, Ouanes JP. Seldinger technique for nasal intubation: a case series. Journal of Clinical Anesthesia. 2016; 34: 609611.

[4] Elwood T, Stillions DM, Woo DW, Bradford HM, Ramamoorthy C. Nasotracheal intubation: a randomized trial of two methods. Anesthesiology. 2002; 96: 51-53.

[5] Delgado AV, Sanders JC. A simple technique to reduce epistaxis and nasopharyngeal trauma during nasotracheal intubation in a child with factor IX deficiency having dental restoration. Anesthesia and Analgesia. 2004; 99: 1056-1057.

[6] Kim YC, Lee SH, Noh GJ, Cho SY, Yeom JH, Shin WJ, et al. Thermosoftening treatment of the nasotracheal tube before intubation can reduce epistaxis and nasal damage. Anesthesia and Analgesia. 2000; 91: 698-701.

[7] Katz RI, Hovagim AR, Finkelstein HS, Grinberg Y, Boccio RV, Poppers PJ. A comparison of cocaine, lidocaine with epinephrine, and oxymetazoline for prevention of epistaxis on nasotracheal intubation. Journal of Clinical Anesthesia. 1990; 2: 16-20.

[8] Hall CEJ, Shutt LE. Nasotracheal intubation for head and neck surgery. Anaesthesia. 2003; 58: 249-256.

[9] Kim Y. Complications associated with orthognathic surgery. Journal of the Korean Association of Oral and Maxillofacial Surgeons. 2017; 43: 3-15.

[10] Kim H, Yun K, Kim K, Kang H, Choi Y. Computed tomography evaluation and pretreatment for a safe nasotracheal intubation, avoiding nasal cavity injuries. Journal of the Korean Association of Oral and Maxillofacial Surgeons. 2010; 36: 197. (In Korean)

[11] Adamson DN, Theisen FC, Barrett KC. Effect of mechanical dilation on nasotracheal intubation. Journal of Oral and Maxillofacial Surgery. 1988; 46: $372-375$

[12] Buchanan EP, Hyman CH. LeFort I osteotomy. Seminars in Plastic Surgery. 2013; 27: 149-154.

[13] Movahed R, Morales-Ryan C, Allen WR, Warren S, Wolford LM. Outcome assessment of 603 cases of concomitant inferior turbinectomy and Le Fort I osteotomy. Proceedings. 2013; 26: 376-381.

[14] Song I, Choi J, Baik U, Ryu J, Lim J, Choi Y, et al. Recovery pattern following bimaxillary orthognathic surgery: Differences between sexes. Journal of Cranio-Maxillofacial Surgery. 2019; 47: 138-142.

How to cite this article: Je Jin Lee, Geun Joo Choi, Hyun Kang, Chong Wha Baek, Su Hyun Seo, Young-Jun Choi, et al. Le Fort I osteotomy facilitated nasotracheal intubation in a patient with concha bullosa and septal deviation: a case report. Signa Vitae. 2021;17(3):238-241. doi:10.22514/sv.2021.062. 